\title{
escrito porna.
}

'Jelitza Soto-Román

Profesora y coordinadora de Práctica Supervisada, Universidad del

Sagrado Corazón. Bachillerato en Artes, concentración en Trabajo

Social. Maestría en Trabajo Social con Familias, Niños y Adolescentes.

Disertación doctoral en proceso (psicología clínica). Universidad

de Puerto Rico, Río Piedras.

$\triangle$ jelitza.soto@sagrado.edu

(D) ORCID ID https://orcid.org/0000-0002-2785-1230

Cómo citar / citation: Soto-Román, J. (2020). La formación profesional del Trabajo Social en Puerto Rico ante el contexto actual: Desafíos y posibilidades. Voces desde el Trabajo Social, 8(1), 176-199. https://doi.org/10.31919/voces.v8i1.223

Recibido / received:

4 de septiembre de 2020
Revisado / reviewed:

28 de octubre de 2020
Aceptado / accepted: 8 de diciembre de 2020

Derechos de autoría / Copyright: (c) 2020 Soto-Román, J. Este es un artículo de acceso abierto y distribuido bajo los términos de la licencia y políticas de Creative Commons Attribution 4.0 International License. 


\section{LA FORMACIÓN LA PROFESIONAL DEL TRABAJO SOCIAL EN PUERTO RICO ANTE EL CONTEXTO ACTUAL: DESAFÍOS Y POSIBILIDADES}

Resumen

fi OPENACCESS PEER-REUEWED

El contexto socio-económico y político que actualmente permea nuestra sociedad puertorriqueña, atraviesa la vida de todos y todas como ciudadanos/as y seres humanos. Una crisis económica que pareciera no tener resolución, condiciones de vida precarias, derechos vulnerados, entre otras realidades que lamentablemente caracterizan la realidad que vivimos. Todo esto tiene como telón de fondo un contexto neoliberal que ha venido exacerbando cada vez más la realidad de vida de las personas. Como profesión y colectivo, el trabajo social tiene un compromiso con la defensa de los derechos fundamentales y la denuncia de todo aquello que implica su vulneración. No obstante, tanto la profesión como el colectivo que la conforma, no están exentos de ser impactados por esta realidad neoliberal que nos amenaza. Una realidad presente, pero a veces disfrazada, que presenta desafíos a nuestro quehacer profesional y a la consecución del proyecto ético-político que estamos intentando construir en Puerto Rico. Inevitablemente, como un efecto en cadena, el proceso de formación en trabajo social, también se ve atravesado por dicho contexto. Reconociendo este panorama, el siguiente artículo pretende, en primer lugar, propiciar la reflexión sobre la formación profesional en trabajo social. Posteriormente, exponer algunos de los retos que representa el contexto actual 
para la educación superior en general, y la formación profesional en particular. Por último, ante la discusión y análisis de dichos retos, proponer acciones o alternativas que posibiliten sostener una formación profesional que trascienda los desafíos que enfrentamos en los distintos ámbitos del trabajo social.

\section{Palabras claves}

trabajo social, formación profesional, educación superior, contexto neoliberal

\section{Sumario}

Forma-ción profesional y Trabajo Social. La educación superior en Puerto Rico y el Trabajo Social. Retos para la formación profesional en Trabajo Social. Posibilidades desde el Trabajo Social para la formación profesional. Referencias.

\section{[EN] SOCIAL WORK PROFESSIONAL FORMATION IN PUERTO RICO AND THE CURRENT CONTEXT: CHALLENGES AND POSSIBILITIES}

\section{Abstract}

The socio-economic and political context that currently pervades our Puerto Rican society impacts the lives of all of us as citizens and human beings. An economic crisis that seems to have no resolution, precarious living conditions, human rights violations, among other realities that unfortunately characterize the reality we live in. All this has, as a backdrop, a neoliberal context that has been increasingly exacerbating the reality of people's lives. As a profession and collective, social work has a commitment to the defense of fundamental rights and the denunciation of everything that implies their violation. However, both the profession and the collective are not exempt from being impacted by this neoliberal reality that threatens us. This reality, sometimes disguised, presents challenges to our professional work and to the achievement of 
the ethical-political project that we are trying to build in Puerto Rico. Inevitably, as a chain effect, the social work education and training processes are also crossed by that context. Recognizing this picture, the following article aims to encourage reflection on educational and professional training in social work. Subsequently, set out some of the challenges that the current context represents for higher education and professional formation. Finally, in the face of the discussion and analysis of these challenges, propose actions or alternatives that make it possible to sustain a professional formation that transcends the challenges we face in different areas of social work.

\section{Keywords}

social work, professional formation, higher education, neoliberal context

\section{Introducción}

"Enseñar, es enseñar a dudar."

- Eduardo Galeano

"Creo que cada vez menos tiene que ver con los contenidos y cada vez más con provocar un acontecimiento educativo que es otra cosa, que es inspirar a que los estudiantes busquen su propia transformación."

-Darío Sztajnszrajber

Quiero comenzar compartiendo y resaltando que el desarrollo de las reflexiones que se presentan en este escrito no son solo mías, pues son muchas las personas que contribuyeron a lo que ha terminado plasmado en estas letras. Más allá de las autoras y autores que consulté, debo decir que también aportaron mis estudiantes, desde esas discusiones sostenidas en y fuera del salón de clases. Contribuyeron además mis colegas, docentes y no docentes, y no solo del ámbito del trabajo social, sino del campo interdisciplinario. Particularmente desde esas reflexiones sobre lo que está ocurriendo en el país, en la educación superior, sobre los retos que estamos enfrentando. También sobre las estrategias que en el camino hemos ido delineando para defender espacios y crear las condiciones de la educación en la que creemos y del proceso de formación que queremos facilitar y sostener. Por último, me parece importante destacar que para el desarrollo de este artículo hice el 
esfuerzo consciente de considerar lo que han escrito colegas en Puerto Rico respecto a varios de los temas que estaré abordando. No obstante, reconociendo que los mismos también han sido trabajados en otros países de América Latina y el Caribe, estaré integrando algunas puntuaciones sobre el análisis de estos temas desde otros contextos. Mi reconocimiento y agradecimiento a todas y todos por inspirar y nutrir algunas de estas reflexiones.

\section{Forma-ción profesional}

\section{y Trabajo Social}

Al detenernos en el concepto de forma-ción, es posible identificar que la palabra forma viene del latín forma, que remite a una figura o imagen; $y$ el sufijo latino ción, que indica acción $y$ efecto. Digamos entonces que formación implica tanto la acción como el efecto de formar o dar forma a algo. Considerando esto, cuando hablamos de formación profesional se nos presenta la complejidad de aquellas acciones necesarias $o$ requeridas en la búsqueda de dar forma o alcanzar el efecto de formar la figura o imagen de un profesional. Ahora bien, ese proceso, que por remitirse a la acción denota que es dinámico y que implica un resultado, se complejiza aún más cuando hablamos de formar profesionales en el campo del trabajo social. ¿De qué se tratan esas acciones? ¿Cuál es la imagen o figura de ese profesional que se busca formar? ¿Es única y uniforme? ¿Quién determina eso? Estas y otras preguntas, son las que me gustaría poner en perspectiva durante este escrito.

A mi entender habría que comenzar por preguntarnos, ¿de qué hablamos cuando hablamos de formación profesional? Para comenzar, podemos hacer alusión a los procesos de formación legitimados y formales. En primer lugar, los programas académicos a nivel graduado y sub-graduado, junto a su profesorado, quienes tienen a cargo la importante responsabilidad de formar, o mas bien proveer los primeros "pininos" de formación para profesionales del trabajo social. En segundo lugar, podemos pensar en la formación profesional como un proceso que interpela también a quien es colocado en el lugar de aprendiz. ¿Qué quiero decir con esto? Que el proceso de formación profesional no se trata de una tarea como la del alfarero que da forma a una vasija, siendo la vasija el/la estudiante aprendiz. Si tomamos esta metáfora, no podemos perder de perspectiva que el barro es un material pasivo y maleable, y que son las manos del alfarero las que le dan la forma elegida por este. Sin embargo, cuando se trata de seres humanos, ni estamos hablando de entes pasivos y siempre maleables, 
ni la tarea de "dar forma" descansa solo en las manos de a quien se reconoce como aquel (o aquella) que tiene el conocimiento y la experiencia. Tal como asevera uno de los epígrafes que acompaña este escrito: "cada vez menos tiene que ver con los contenidos y cada vez más con provocar un acontecimiento educativo que es otra cosa, que es inspirar a que los estudiantes busquen su propia transformación".

Siendo así, cabría decir que cuando hablamos de formación profesional, no podemos reducirla a un proceso deenseñanza-aprendizaje cuya responsabilidad recae sobre una sola de las partes implicadas en el proceso: el profesorado, el estudiantado, los programas académicos, las universidades. Por el contrario, hablar de formación profesional implica un proceso complejo, continuo, compartido y cambiante, que comprende varios personajes y elementos. Entre ellos, los más evidentes y ya mencionados, aquellos y aquellas a quienes se les atribuye la tarea principal de formar profesionales del trabajo social para que ejerzan o practiquen en los distintos escenarios donde se necesita o espera nuestra labor. Sin embargo, si concebimos la formación profesional como un proceso sostenido de pensar nuestro trabajo, remitirnos a las teorías, cuestionar la realidad, aprender continuamente; tendríamos que decir entonces que más que un requerimiento que nos capacita para ejercer como profesionales del trabajo social, es condición de posibilidad para que nuestra profesión pueda ser y se sostenga.

Partiendo de esta reflexión, tendríamos que poner en perspectiva preguntas como: ¿De qué hablamos cuando hablamos de formación profesional en trabajo social? ¿Qué representa la formación para una profesión como el trabajo social? A mi manera de ver, la formación del profesional del trabajo social implica primordialmente un posicionamiento ético-político que trasciende el aprendizaje de contenidos y el desarrollo de destrezas. $Y$ cuando digo que trasciende, no quiero decir que tiene poca importancia; sino que las áreas teóricometodológicas y técnico-operativas son fundamentales, pero que sin el componente ético-político, terminan siendo lo que es requerido a cualquier otro profesional de cualquier otro campo o disciplina. Ahora bien, desde mi perspectiva, esta es una de las dimensiones más complejas y que mayor dificultad presenta para todas las partes implicadas.

Quiero aclarar que cuando hablo de las partes implicadas pienso en las escuelas y programas de formación profesional a nivel graduado y sub- 
graduado en Puerto Rico y, con ellas, en la facultad y estudiantes que las conforman. Pienso además en nuestro Colegio de Profesionales del Trabajo Social y su Instituto de Educación Continuada; pues si consideramos la formación como un proceso continuo de capacitación y reflexión, no podemos limitarla a la obtención de un grado académico o profesional. Finalmente, pienso en aquellos y aquellas colegas que trabajan en los escenarios donde se da la integración teoría y práctica, quienes posibilitan el aprendizaje en contexto de futuros/ as colegas. Esos/as que conocen de primera mano los retos que implica sostener una formación y práctica acorde con la filosofía y principios de nuestra profesión. Teniendo esto como trasfondo, me parece entonces importante detenernos brevemente en algunos elementos sobre la educación en trabajo social, considerando el contexto actual de la educación superior en Puerto Rico.

\section{La educación superior en}

\section{Puerto Rico y el Trabajo Social}

La educación ha sido fundamental para la profesión de trabajo social desde sus inicios en nuestro país; no es resultado del azar que en un primer tiempo se les llamara "maestras visitantes". En la actualidad, la educación en trabajo social en Puerto Rico se ofrece mayormente por instituciones privadas $(79 \%=11$ instituciones). Existen 14 instituciones de educación superior que ofrecen un total de 19 programas subgraduados y graduados de Trabajo Social. De estos, 13 son programas sub-graduados y seis son programas graduados (ANAETS, 2018). Once (11) de las 14 instituciones (64\%) y 13 de los 19 programas (68\%), o sea la mayoría, están asociados a la Asociación Nacional de Escuelas de Trabajo Social de Puerto Rico, conocida por sus siglas como ANAETS.

La educación y formación profesional del trabajo social en Puerto Rico, ha estado influenciada desde sus inicios por las escuelas de formación norteamericanas a partir de la relación colonial con los Estados Unidos que aún prevalece al día de hoy. Según Cordero (2011), "la relación colonial que tiene Puerto Rico con los Estados Unidos de América ha llevado a que no se tomen en consideración los aspectos culturales al regular la formación y la práctica profesional” (p. 8). Esto se ha traducido en una formación determinada por instancias ajenas a nuestra realidad socio-cultural; lo que a su vez implica que nosotras y nosotros mismos/as, hemos quedado enajenados/as del proceso de elegir el trabajo social que queremos para nuestro país. Estas circunstancias han planteado retos particulares 
a los procesos de formación en Puerto Rico, al igual que paradojas y contradicciones para la práctica del trabajo social. Sin embargo, el contexto socio-económico y político en el que vivimos actualmente, presenta nuevos desafíos a una formación que históricamente ya viene lidiando con retos que aún no han sido superados del todo.

La complejidad de la situación socio-económica y política de Puerto Rico nos presenta un sinnúmero de retos ante un contexto caracterizado por lo siguiente: aumento en todas las manifestaciones de la violencia, creciente pobreza y desigualdad social que surge de la creación y acumulación de riqueza de parte de unos sectores mediante el establecimiento de políticas neoliberales; cambio demográfico acelerado por la emigración masiva, medidas de austeridad impuestas por la Junta de Control Fiscal que agravan el desmantelamiento del sector público, entre muchas otras realidades. Estos desafíos se manifiestan directamente en el ámbito de la educación superior, donde como ya he planteado, se dan los inicios y fundamentos de la formación profesional. La educación superior en Puerto Rico, atravesada también por un contexto neoliberal, ha sufrido una serie de cambios que han tenido implicaciones en los distintos programas de formación.
De manera particular, estos cambios han sido más acelerados y evidentes en la segunda década del siglo $\left.X X\right|^{8}$. Los cambios e implicaciones a los que me refiero, van desde elementos tan escuchados como los recortes en presupuesto, hasta un cambio radical en las direcciones que se propone dar a la educación y formación universitaria.

Según la Dra. Carmen Dolores Hernández (2019), educadora, la educación superior en Puerto Rico se devalúa cada vez más en aras de propósitos que poco tienen que ver con la enseñanza y la capacitación profesional: se bajan las exigencias académicas y sufre la calidad de la enseñanza. Manifestaciones de esto se observan en el énfasis de algunas instituciones en la formación técnica y la reducción de la formación en artes liberales. A su vez, se ve amenazado el desarrollo de pensamiento crítico que posibilita el cuestionamiento de la realidad social y la toma de acción sobre esta para transformarla. Por otro lado, las políticas neoliberales y la flexibilización de los derechos laborales que han traído consigo, han propiciado que cada vez más, las universidades funcionen a partir de la contratación de facultad a tarea parcial con un mínimo de

8 Aceleramiento que se concretó a principios del 2020 con el movimiento hacia la educación a distancia ante las medidas de control y prevención impuestas mundialmente para el manejo de la pandemia por el COVID-19. 
facultad a tarea completa. La apertura de "ventanas" de retiro y el congelamiento de plazas regulares o permanentes, viabiliza y solidifica la contratación de mayor cantidad de facultad a tarea parcial.

Según la Asociación Nacional de Escuelas de Trabajo Social en Puerto Rico (2018), el aumento en la contratación de docentes a tiempo parcial, contribuye a la precarización de las condiciones laborales de los y las docentes. Demetrio (2016) lo describe como "la sustitución degradada del trabajo docente con titulación por el profesional especialista que, sin estabilidad y con un salario más bajo, se adapta a los intereses de las empresas que actúan en el mercado de la enseñanza superior" (p. 86). Esto representa serios retos para los programas académicos $y$, sobre todo, para aquellos/as colegas bajo este tipo de contratos, pues en cierta medida se atenta contra su derecho a tener condiciones de trabajo dignas y una estabilidad económica que se ve amenazada semestre a semestre según transcurre el año académico. El aumento en la contratación de profesoradoencondiciones detrabajo poco favorables, puede influenciar incluso la dinámica de trabajo dentro de un programa académico. Como menciona Garriga (2018) respecto a la educación pública en Puerto Rico, estamos hablando de "la creación de un panorama desolador en materia de derechos laborales y garantías de empleo" (p. 188).

Por otra parte, las administraciones universitarias buscan maneras de lidiar con el impacto del contexto económico y la emigración, lo que mayormente se ve reflejado en la disminución de estudiantes que se matriculan. Una quinta parte del cuarto de millón de estudiantes que había a comienzos de la década (2010-2019) en instituciones post secundarias puertorriqueñas, ha ido desapareciendo (Aragunde, 2019). El éxodo de población hacia Estados Unidos, trae como consecuencia la disminución de matrícula en todos los niveles del sistema de educación. Esto es particularmente cierto para la educación superior, comprometiendo a las instituciones públicas y privadas a la reducción de personal docente y los beneficios marginales que devengan; así como a una re-estructuración programática y administrativa que limita la oferta académica y los servicios al estudiantado y docentes (ANAETS, 2018). Como consecuencia de esto, tal como plantea Hernández (2019), "las burocracias administrativas olvidan a menudo los fines para los que surgieron y enfocan sobre los medios para mantenerse en sus puestos [...] La educación se convierte en un negocio rentable con 'clientes' cautivos." (p. 1). Esto se ve reflejado en el alto 
costo de la educación privada y en los aumentos en los costos de matrícula en el sistema público. La colega mexicana Ornelas (2019) comparte este análisis al señalar que: "la educación considerada como mercancía se ha convertido en un negocio, en una transacción, lejos de la idea inicial de la educación como proceso de formación de sujetos y como derecho fundamental" (p. 154).

Según el Informe sobre Desarrollo Humano en Puerto Rico (2016), el $79 \%$ de las instituciones universitarias en el país pertenece al sector privado y reciben entre el $85 \%$ y 95\% de sus ingresos de los pagos de matrícula de sus estudiantes; quienes a su vez, reciben la mayor parte del dinero a través de becas o préstamos del gobierno federal. De acuerdo a los/as autores/as de este Informe (2018), algunos estudiosos consideran esto un factor que "hace parecer que la situación económica de la población estudiantil universitaria es favorable, cuando en realidad muchos provienen de familias pobres y pueden pagar sus estudios solo gracias a estas ayudas" (p. 169). De hecho, muchos estudiantes que logran completar un grado académico, lo hacen a base de muchos sacrificios, y en ocasiones manteniendo uno y dos trabajos para poder completar el pago de sus estudios y el ingreso mínimo que cubra sus necesidades.
De acuerdo a Chomsky (2014), esto forma parte de la lógica empresarial neoliberal que se ha trasladado a las universidades, y cumple además la función de "adoctrinar a los jóvenes", cargándolos con deudas pesadas para poder sufragar sus estudios.

Cabe destacar que las implicaciones de las políticas neoliberales en la educación superior discutidas hasta el momento, se manifiestan de manera similar en otros países de América Latina y el Caribe. Basta y Cavalleri (2019) presentan una atinada radiografía de estas implicaciones en países como Argentina, Bolivia, Brasil, Chile, Paraguay, Perú y Uruguay. Estas colegas se dieron a la tarea de realizar un estudio de ponencias presentadas en los últimos dos Seminarios de la Asociación Latinoamericana de Enseñanza e Investigación en Trabajo Social (ALAEITS); celebrados en Córdoba, Argentina en el año 2012, y en Mazatlán, México en el año 2015. En su análisis abordan el tema de la formación profesional en trabajo social y sus vinculaciones con las políticas deeducación superior a partir de la influencia neoliberal. Aunque no de manera exhaustiva, señalan tendencias presentes en las políticas de educación superior planteadas en las ponencias estudiadas, entre las que se destaca la mercantilización del proceso educativo. No obstante, enfatizan que "el impacto de las 
políticas neoliberales tendrá expresiones diferenciales" en cada país (Basta y Cavalleri, 2019, p. 14). Este es el caso de nuestra experiencia en Puerto Rico, donde la situación colonial impregna estas implicaciones de paradojas y retos particulares.

Un ejemplo de esto es que en nuestro país se ha incrementado, por un lado, la oferta y por el otro, el interés de parte de las instituciones universitarias en responder a estándares de acreditación y a paradigmas de formación e investigación establecidos por entes externos. En el caso de Puerto Rico, no debe extrañarnos que estas instancias externas sean en su abrumadora mayoría entidades estadounidenses: Middle State Comission on Higher Education (MSCHE), American Psychological Association (APA), Council on Social Work Education (CSWE), entre otras. Respecto a esta última, actualmente en Puerto Rico casi el total de los programas de formación en Trabajo Social, tanto a nivel graduado como sub-graduado, están acreditados por dicho organismo. Esto resulta interesante, ya que para ejercer la profesión en Puerto Rico, no es necesario que el grado académico que se requiere para obtener la licencia sea de una universidad acreditada por este organismo. Sin embargo, en la mayoría de los programas y escuelas de formación en Puerto Rico, desarrollamos y enseñamos currículos a partir de lo que se establece en los estándares de acreditación de esta agencia reguladora.

En relación a esto, colegas de distintos programas que forman parte de la ANAETS, y que participaron de un ejercicio de exploración desarrollado para fines de este escrito, señalaron el reto que representa atemperar el currículo a las corrientes de trabajo social contemporáneo debido a las particularidades de la agencia acreditadora y al poco espacio para enseñar fuera del modelo generalista a nivel sub-graduado (Soto, 2019). No obstante, también indicaron que ante las dificultades y reducción de recursos económicos que enfrentan actualmente muchos programas académicos, los procesos de acreditación se convierten en una estrategia para lograr que las administraciones universitarias destinen recursos para el funcionamiento efectivo de estos. Digamos entonces, que más allá de la tendencia histórica que vincula el trabajo social en Puerto Rico al trabajo social en los Estados Unidos, también es necesario reconocer que, paradójicamente, el ser un programa acreditado denota cierto prestigio o posición en el contexto de la educación superior, ya sea en instituciones públicas o privadas. Es así que, en cierta medida, la acreditación 
garantiza reconocimiento, determina otorgación de presupuesto y define asignación de recursos; entre otros aspectos necesarios para sostener un proceso de formación profesional dentro de un contexto universitario.

Ahora bien, más allá de los beneficios o ganancias que sería posible argumentar respecto a las funciones de regulación y establecimiento de estándares de calidad que estos organismos cumplen, no es menos cierto que también representan lo que Rivera (2019) llama la "penetración de la lógica neoliberal en el espacio académico" (p. 96). Con esto hace referencia a cómo, organismos que ostentan cierto poder legitimado de ser garantes de la calidad o excelencia de los programas e instituciones de educación superior, responden igualmente a una agenda neoliberal que privilegia ciertos paradigmas de investigación en la producción del conocimiento. Un ejemplo de esto es abordado por Román (2016, parr. 2) cuando señala que:

Detrás del avance de la llamada investigación basada en evidencia se encuentra todo un entramado de poder que, encarnado en las agencias acreditadoras de corte profesionista (psicología, trabajo social, administración pública, por ejemplo), va colonizando no solo las agencias de gobierno locales sino el mundo de la academia.
Según su análisis, dicho establecimiento de lo académicamente correcto a partir de lo que es posible evidenciar como algo que funciona para todos los que comparten una condición, problemática, síntoma o situación de vida, atenta contra la libertad de cátedra e intensifica el trabajo del profesorado, amenazando incluso la permanencia o existencia de departamentos o programas académicos.

\section{Retos para la formación profesional en Trabajo Social}

Este panorama descrito, que puede parecer amenazante $y$ desolador, es precisamente el que enfrenta la mayoría, sino todos, los programas académicos de formación en trabajo social en Puerto Rico. Desde la reactivación de la Asociación Nacional de Escuelas de Trabajo Social en Puerto Rico (ANAETS), esta organización ha realizado esfuerzos por generar espacios de encuentro y reflexión entre distintos programas y escuelas de formación en trabajo social que son integrantes de la misma. Esta organización comienza en el 1998, pero se reorganiza en el 2010, estableciendo como visión la aspiración por una educación en Trabajo Social competente y crítica, que contribuya al desarrollo de una sociedad justa y protectora de los derechos humanos (ANAETS, 2012). Como se mencionó anteriormente, 
la mayoría de escuelas y programas de Trabajo Social en Puerto Rico, forman parte de esta asociación, así como más de 12 miembros individuales, estudiantes y otras organizaciones afiliadas, como el Colegio de Profesionales del Trabajo Social de Puerto Rico (CPTSPR).

En el transcurso de los años, han sido varias las instancias en las que esta organización ha planteado la necesidad de reflexión y acción ante el contexto actual que atraviesa la formación profesional en trabajo social. En el año 2012, a dos años de haberse reorganizado, tuve la oportunidad de participar como representante de dicha organización en el Foro de formación subgraduado que se llevó a cabo en el XX Seminario Latinoamericano de Escuelas de Trabajo Social celebrado en Argentina. Allí, se compartieron los retos que enfrentaban las instituciones educativas en Puerto Rico con colegas de otros países. De manera particular, se reconocía la proliferación de los ofrecimientos académicos en el contexto de políticas neoliberales y los retos que esto representaba para sostener una educación de excelencia, tanto en el sistema público, como en el privado. Temas como este se continuaron trabajando en el Seminario XXI que se llevó a cabo en el 2015 en México, donde se plantearon retos similares por parte de escuelas y programas de trabajo social en distintos países. En su análisis, Basta y Cavalleri (2019) los agrupan en tres grandes ejes: cuestiones de "carácter políticoacadémico y gremial" (condiciones de trabajo de docentes y de condiciones de estudio del estudiantado), referencias y reflexiones acerca de los "fundamentos teórico metodológicos" (finalidades de la formación, revisión de planes de estudios, teoría y práctica) y "operacionalización de propuestas y experiencias" de cursos y proyectos de investigación (pp. 18-19).

En el caso de Puerto Rico, para el 2012 se esbozaba como uno de los mayores retos para la ANAETS y los docentes de los programas que la conformaban, el articular una formación en Trabajo Social Crítico a nivel nacional, que incorporara las categorías histórico-críticas y las competencias que requiere un proyecto ético-político profesional, que respondiera a nuestra realidad socio-histórica, en el marco del mercado de la educación y de los requerimientos del CSWE. Cabe señalar que, al día de hoy, esto continúa siendo un reto. Sin embargo, resulta importante destacar que según señalan algunos/as colegas (Soto, 2019), aunque no hemos enfrentado con mayor tenacidad los requerimientos del CSWE que no atienden nuestra realidad sociocultural, a través de la ANAETS se 
han logrado negociar elementos y procesos para que respondan al contexto puertorriqueño. Para este año, la mayor parte de los programas tenía una matrícula entre los 100 y 800 estudiantes, la plantilla de personal docente a tiempo completo era de entre uno (1) a cinco (5) docentes en el 62.5 por ciento de los programas (ANAETS, 2012). Por lo cual, todos los programas tenían la necesidad de contratar personal docente a tiempo parcial para distribuir la carga académica de los programas.

Como mencioné anteriormente, en el contexto actual de la educación superior, esta práctica continúa. De hecho, se ha incrementado el congelamiento de plazas a tiempo completo y la sustitución de estas por contratos temporeros o parciales. Son muchos los programas que han expresado la necesidad de poder contarconmás profesores/asatiempo completo que pudieran ser parte de todos los procesos académicos y contribuir al fortalecimiento de los procesos educativos. Esto presenta preocupación por el hecho de que, en algunos programas, la facultad a tarea parcial es mayor a la cantidad de facultad a tarea completa, lo que puede afectar la coherencia y consistencia del diseño curricular. Al mismo tiempo, se plantea el reto de cómo integrar y vincular esta facultad a los procesos institucionales sin ser opresores/as, considerando las condiciones de trabajo en las que estos/as se encuentran (Soto, 2019). En relación a las condiciones laborales del personal docente, la única área que en aquel momento fue señalada como una de mucha satisfacción, fue la libertad de cátedra. Asunto que como también se mencionó, en la actualidad se está viendo amenazado por el empuje hacia paradigmas de investigación y enseñanza uniformes (Román, 2016). Cabe señalar que, para aquel entonces se entendía que había una gran disparidad entre el sector público y el privado; en la medida que en el primero había oportunidad para plazas permanentes, mejores salarios y beneficios marginales, así como participación en la toma de decisiones. No obstante, esto ha cambiado de forma dramática en los últimos años a raíz del impacto de las políticas neoliberales en el sector público.

Desde mi perspectiva, y la de muchos/as colegas con quienes he tenido la oportunidad de intercambiar impresiones al respecto, hoy día, los retos que se identificaron en aquel entonces continúan presentes, y otros se han convertido en desafíos más complejos. Tan reciente como en el 2018, un grupo de colegas de la ANAETS estuvo participando del XXII Seminario Latinoamericano y Caribeño de Escuelas de Trabajo Social celebrado en Bogotá, 
Colombia. Allí se expresó la necesidad inminente de fortalecer la capacidad política para incidir de manera efectiva en las instituciones en las que se forman profesionales del trabajo social (ANAETS, 2018). Según el informe desarrollado a partir de la participación en dicho Seminario, la educación de trabajo social en Puerto Rico enfrenta un desafío histórico en la articulación entre los proyectos académicos y profesionales, particularmente relacionados a la investigación. Uno de los mayores obstáculos que, de acuerdo a este informe, enfrentan las unidades académicas para la investigación en los contextos universitarios, son las políticas neoliberales. Las manifestaciones de estas políticas se reflejan en la tendencia a la mercantilización y privatización de la educación, transformando los procesos de formación, reduciendo el gasto y aumentando los resultados que respondan a respuestas inmediatas a los asuntos que surgen de las cuestiones sociales (ANAETS, 2018).

Esta demanda de inmediatez en las respuestas, se traduce a su vez en el requerimiento de una educación igualmente inmediata; entiéndase, completar un grado académico en el menor tiempo posible. Esto ha llevado a la revisión y desarrollo de programas a nivel graduado y subgraduado que, en aras de competir, proponen ofertas de programación para agilizar la terminación del grado en menos tiempo. Lo mismo ocurre en México y otros países latinoamericanos, donde se ha observado una proliferación de programas de estudios de corta duración, tanto a nivel graduado como sub-graduado; reduciendo así el tiempo de formación y limitando el proceso de enseñanza al entrenamiento y la posterior incorporación del egresado/a al mercado laboral (Demetrio, 2016; Ornelas, 2019). Estas propuestas o diseños curriculares, por lo general, no están concebidas en criterios pedagógicos o de formación profesional, sino en propuestas de tipo economicistas. Como plantea Batalla Plá (2008), "pareciera necesario demandar al Trabajo Social únicamente tareas instrumentales y soluciones de corto alcance, que no pongan a la vista las contradicciones del orden burgués y que por tanto no perjudiquen su funcionamiento y su incesante búsqueda por mantener el régimen de acumulación" (p. 56).

Esto nos plantea la pregunta sobre ¿a qué criterios o exigencias está respondiendo actualmente la educación en trabajo social?: ¿al mercado, a las agencias acreditadoras, al gobierno, a las administraciones universitarias? De repente, sorprendería percatarnos del riesgo inminente de responder 
a las demandas de muchos otros, excluyendo las nuestras y las que nos imponen nuestros principios éticos. Parecería ser entonces que corremos el peligro de que otros terminen definiendo qué es el trabajo social, qué se espera de un profesional de ese campo, cómo se debe enseñar la profesión, hacia dónde debe dirigirse, y a qué propósitos o intereses responde. Es así que enfrentamos el reto, no solo de defender la forma en que entendemos se debe enseñar y ejercer la profesión; sino que estamos frente a un desafío aun mayor, ante la pretensión arrogante de otros en tomar decisiones sobre ella. Desde mi perspectiva, si no nos detenemos a reflexionar sobre estas contradicciones, tendremos que asumir un costo muy alto. Pues entre el establecimiento de parámetros que nada tienen que ver con nuestros principios, y el intento de otros en re-nombrar y desarticular la profesión, podemos caer en la trampa de la sobrevivencia. Esa sobrevivencia tan característica del proyecto capitalista neoliberal con sus propuestas de revitalización de la competencia en el mercado y la reconstrucción y apuesta por el individualismo (Batalla, 2008). Si nos concentramos solo en el intento de sobrevivir, como persona, como programa, como institución, como profesión, terminaremos cayendo en la trampa y el resultado será desaparecer o perder nuestra identidad y convertirnos en otra cosa.

Finalmente, otro de los retos que enfrentan los programas académicos en trabajo social remite a una de las áreas más importantes dentro del proceso de formación en las universidades: el estudiantado. A diferencia de décadas anteriores, en las cuales el estudiantado por lo general estudiaba a tiempo completo y rara vez trabajaba, en la actualidad todos los programas tienen un cuerpo estudiantil que en su mayoría trabaja (ANAETS, 2012; Soto, 2019). La realidad que vive el estudiantado que llega a nuestros programas está mediada también por el contexto socio-económico que les obliga a trabajar mientras estudian. De hecho, en ocasiones, del ingreso de ese trabajo no solo depende el/la estudiante, sino que muchas veces son ellos y ellas quienes corren con la mayor parte de los gastos económicos en sus familias. Esto tiene repercusiones en el tiempo que pueden dedicarle a sus estudios, e incluso en el nivel de energía o interés con el que llegan a la sala de clases. Muchas veces cansados/as o trasnochados/as por los turnos de trabajo que tienen que asumir. Es así como en las aulas, en los pasillos y en las oficinas de los programas de formación, también nos enfrentamos a un panorama en el que son palpables los efectos de las políticas 
neoliberales. La precariedad de las condiciones sociales, económicas y laborales se reflejan en la vida de aquellos/as que desean convertirse en profesionales para, irónicamente, trabajar con esa misma realidad.

Es en esta lógica neoliberal omnipresente y amenazante que se nos plantea la encomienda y el reto de educar en trabajo social, de hacer trabajo social; de fungir como profesionales del trabajo social. Sin embargo, más allá del panorama descrito y de los retos y desafíos planteados, según la Encuesta Mundial de Valores para Puerto Rico 2018 (Hernández, 2018), de un total de 26 tipos de instituciones, las universidades son las que generan un mayor nivel de confianza (79.8\%) entre las personas en Puerto Rico. Desde mi perspectiva, esta confianza que aún se sostiene en la educación superior, es posible interpretarla como algo que infunde esperanza, pero al mismo tiempo, como una gran responsabilidad. Sobre todo, para aquellos y aquellas que asumimos roles y funciones en las diversas instituciones universitarias. Con esto como referente, y asumiendo una responsabilidad esperanzadora, pretendo culminar mi exposición discutiendo algunas propuestas, acciones 0 alternativas que han surgido de las consultas, diálogos, lecturas, trabajos y reflexiones sostenidas con colegas y estudiantes. Las comparto con la intención de que puedan traducirse en posibilidades de sostener la formación y práctica de un trabajo social integrador $y$ sólido ante una embestida neoliberal que pretende acorralarlo y silenciarlo.

\section{Posibilidades desde el Trabajo Social para la formación profesional}

Ante un contexto caracterizado por una vorágine de demandas y responsabilidades, resulta necesario hacer pausas y detenernos a reflexionar. La colega argentina Malacalza (2003) considera fundamental el debatir interrogantes sobre las tendencias que se perfilan, tanto en las demandas laborales como en las exigencias de formación profesional. Según lo concibe, esta es una decisión impostergable si no queremos quedar excluidos como sujeto colectivo profesional; y a estos efectos, propone dos movimientos, uno de continuidad y otro de ruptura:

El de continuidad está dado por la preservación de los rasgos de la identidad profesional construidos hasta el momento, y el de ruptura, por la aprehensión de los elementos constitutivos de la nueva cuestión social direccionados desde una perspectiva sustentada en los derechos humanos (Malacalza, 2003, p. 118).

¿Cómo pensar los posibles horizontes y retos que nos plantea 
una perspectiva de derechos humanos? Sobre todo, al reconocer las críticas que se han hecho al discurso de los derechos humanos como una imposición occidental en el contexto neoliberal, o como una trivialización desde lo puramente humanitario (Fontánez, 2020). Ante esto como posibilidad y reto, me parece fundamental el rescate de la dignidad humana y la función política inherente a ellos.

De acuerdo a Rivera (2019), los derechos humanos han sido convertidos en mercancías rentables bajo el nuevo orden económico, convirtiendo a las personas en consumidores y estableciendo un "acceso limitado a personas con el capital suficiente para comprar sus derechos" (p. 95). Algo que resulta sumamente paradójico, pues los derechos que se supone sean reconocidos y garantizados para todo ser humano, ahora son mercancías a las que se tiene acceso de acuerdo a la capacidad económica que tenga la persona. Partiendo de esto, me parece que la invitación sería a remirar-nos como humanos con derechos, y movilizarnos para garantizar que sean respetados; tanto para esos/as con quienes trabajamos, como para nosotras y nosotros como profesionales y humanos. Se trataría de propiciar un contexto en donde no solo se cumplan y defiendan los derechos humanos de las personas, sino uno donde se politicen y amplíen con y desde el trabajo con otros y otras.

Ahora bien, asumir este reto como posibilidad, ameritaría la revisión de los currículos en los programas académicos de formación en trabajo social. Colegas de distintas instituciones universitarias coinciden en que para ello, la actualización de contenidos, los cambios en diseños curriculares y la solidificación de los fundamentos filosóficos de la profesión, resulta fundamental. De igual forma, se trataría de atemperar el currículo a las corrientes de trabajo social contemporáneo, la creación de lineamientos comunes y el desarrollo de áreas de dominio profesional básicas responsivas a nuestro contexto colonial y puertorriqueño (Soto, 2019). Como si se tratara de pequeños pasos hacia un horizonte común, el 18 de octubre de 2019, se celebró el Segundo Encuentro de Escuelas de Trabajo Social de Puerto Rico. Los temas discutidos y las reflexiones allí sostenidas, se tradujeron en un esbozo de posibilidades para la formación en trabajo social desde una perspectiva de derechos humanos. Entre ellas, me parece que una propuesta a destacar sería el abordaje de los derechos humanos desde una perspectiva crítica que sea transversal a los 
currículos. Una que no se reduzca al conocimiento sobre el derecho y los pactos internacionales (teóricometodológico), a una competencia profesional (técnico-operativo), o a un estatuto en el código de ética (ético-político). Esa mirada crítica nos permitiría abordar los derechos humanos no como fin o garantía, sino como posibilidad y apertura.

Otra de las posibilidades que surge de estas reflexiones, es que a pesar de los retos que se enfrentan en las universidades para el desarrollo y la subvención de investigaciones, según la ANAETS (2018), en Puerto Rico existen iniciativas de investigación en Trabajo Social que aportan significativamente al desarrollo de estrategias de acción en pro de la defensa y expansión de derechos humanos, la formación en trabajo social y el desarrollo del proyecto profesional. Para la ANAETS, estas investigaciones aportan también al desarrollo de un currículo de excelencia que tome en consideración la condición política, económica, social y cultural de Puerto Rico. Dichas iniciativas de investigación unen esfuerzos, recursos y docentes investigadores/ as de varias universidades (públicas y privadas), lo que denota el trabajo colaborativo necesario para hacer frente a los desafíos mencionados. Tanto mediante la producción de conocimientos que permitan la consolidación de proyectos, como de la argumentación necesaria para hacer valer nuestros aportes como profesión y colectivo. Además, en ellas también se refleja la vinculación entre la docencia y el gremio profesional (CPTSPR) en los procesos de conceptualización, desarrollo y divulgación de resultados. Vinculación que desde mi perspectiva resulta fundamental para el logro de un proyecto colectivo que, a pesar de su diversidad, pueda sostenerse en el reconocimiento de las diferencias y hacer frente a los desafíos que enfrenta la profesión. De igual forma, estas investigaciones proveen una base para la comunicación y cabildeo en agencias estatales y a nivel legislativo para abogar por mejores condiciones laborales. La propia existencia de la ANAETS y el establecimiento de lazos profesionales con el CPTSPR y otros organismos internacionales, se proyecta como un instrumento de apoyo estructural y conceptual para enfrentar los retos que nos plantea el contexto neoliberal en la educación superior (ANAETS, 2018).

Cabe destacar que, respecto a la investigación, colegas de diversos programas, públicos y privados, coinciden en lanecesidad de fortalecer esta área en la formación profesional. Tanto en la re-integración de esta a 
los currículos para el estudiantado a nivel graduado y sub-graduado, como en las oportunidades 0 condiciones para que el profesorado pueda investigar (Soto, 2019). La colega Rivera (2019) propone la investigación como herramienta socio política para un proyecto de emancipación social desde el trabajo social. Concibe la misma como un acto político que permite re-significar el rolsocial delauniversidad, mediante vínculos de trabajo y alianzas. De igual manera, resalta la importancia del establecimiento de alianzas y colaboraciones con facultad de otras disciplinas que pueda enriquecer los currículos y los procesos de investigación y formación profesional. Colegas de América Latina también coinciden con estas propuestas que consideran la investigación y el establecimiento de alianzas. Se plantea por ejemplo la articulación de docencia e investigación y la producción de conocimientos sobre el trabajo social y la realidad latinoamericana y caribeña (Basta y Cavalleri, 2019; Demetrio, 2016; Ornelas, 2019). De igual forma, se hace énfasis en el enfrentamiento colectivo y articulado, y el fortalecimiento de redes nacionales e internacionales que se constituyan en instancias de intercambio y consulta para las instituciones que forman trabajadores/as sociales (Demetrio, 2016; Ornelas, 2019).
Estrechamente vinculado a la investigación y los currículos académicos, otra área de posibilidad para enfrentar los desafíos en la formación profesional es el fortalecimiento de las relaciones y colaboraciones con los escenarios de prácticaprofesional. Sonesosmismos escenarios, con sus realidades y dificultades, los que proveen los espacios que complementan los currículos académicos con los procesos de práctica supervisada, tanto a nivel graduado como subgraduado. Muchas veces se visualiza a la academia como distante de las realidades que se viven en los escenarios de práctica. Incluso, hay colegas que señalan la existencia de un disloque entre los cursos teóricos, la práctica supervisada y la práctica profesional (Soto, 2019). No obstante, la tan aludida integración teoría y práctica no sería posible sin esos vínculos y colaboraciones. Por tanto, los llamados centros de práctica y los procesos vinculados a la práctica supervisada, deberían representar ese punto de encuentro entre lo teórico-metodológico y lo técnico-operativo. Cabe señalar que en otros países de América Latina y el Caribe también se ha planteado la importancia de la construcción de nexos entre las prácticas de formación de estudiantes, las prácticas profesionales, la docencia 
y la investigación (Basta y Cavalleri, 2019; Ornelas, 2019). No podemos circunscribir la práctica supervisada a un intercambio de recursos, sino reconocer que la misma representa oportunidades de establecer alianzas para el mejoramiento de servicios, el desarrollo de proyectos, la demanda de mejores condiciones de trabajo y el fortalecimiento de la formación profesional; tanto del estudiantado, como de los/as colegas que fungen como colaboradores/as $y$ supervisores/as.

En esta dirección, se trata entonces de cerrar brechas $y$ disminuir distancias entre formación, práctica y gremio profesional. Movernos de la des-vinculación a la vinculación, sin perder de perspectiva que los/as estudiantes son nuestros/as futuros/as colegas. La integración de los/as estudiantes a los procesos vinculados a la investigación, el proyecto profesional y los escenarios laborales mediante la práctica supervisada, contribuye al fortalecimiento de la formación. Además, permite el establecimiento de lazos de solidaridad y trabajo que repercutirán en su posicionamiento y posterior desempeño como colegas del trabajo social. La formación nos compete a todos y todas, se trata de potenciales compañeros/ as de trabajo, de los/as próximos/ as colegiados/as, de la siguiente generación de trabajadores/as sociales que con su práctica nos representarán como profesión. Por tanto, también se trata de quienes ya ostentamos el título de trabajador/a social, de cómo continuamos formándonos como profesionales en distintas áreas. La reflexión sobre nuestro propio proceso de formación siempre debe estar presente; reconocer las rupturas $y$ continuidades que la caracterizan, apropiándonos de ella para hacer frente al contexto actual. De esta forma, si asumimos con voluntad genuina el compromiso de mirar críticamente nuestra formación profesional, estaremos abriendo la posibilidad de que, desde nuestra diversidad, podamos establecer diálogos que permitan esbozar y re-conocer nuestras realidades como profesionales, pero también como colectivo profesional. Se trataría entonces de cómo colaboramos, de cómo sostenemos un trabajo colectivo más allá de las diferencias y contradicciones. De cómo maximizar los espacios de participación y promover incidencias desde la diversidad de contextos y realidades para asumirlos como procesos formativos. La coyuntura histórica que estamos viviendo, es una en la que el trabajo social en Puerto Rico sigue siendo convocado a la reflexión y a la acción; a un 
movimiento que nos permita posicionarnos como profesionales que promueven el cambio y la trans-formación social desde el cuestionamiento, la denuncia y la acción colectiva.

Resulta pues, urgente e inaplazable dejar de visualizarnos como entes fragmentados y aparte. Asumir una consciencia colectiva, construir un nosotros y nosotras. Ser un profesional del trabajo social implica un posicionamiento éticopolítico que debe trascender los espacios y ámbitos de actuación. El horizonte debe ser seguir caminando hacia la articulación de todos los componentes de lo que es la formación profesional en trabajo social. Se han logrado grandes cambios desde las alianzas y vinculaciones que se han destacado; y es importante reconocerlas para no desistir en el enfrentamiento de los grandes desafíos y dificultades que se han ido acrecentando. La apuesta por la creación y el sostén de los lazos sociales, las alianzas y el trabajo colectivo, es desde mi perspectiva la respuesta urgente y necesaria ante los desafíos expuestos. Desde mi propia experiencia, y reconociendo lo compartido por colegas de otras instituciones, es el compromiso y el posicionamiento ético, político y profesional; tanto a nivel personal como colectivo, lo que nos permitirá prevalecer en un contexto que por más que insista en fragmentarlo $y$ desdibujarlo, necesita más que nunca de nuestra perseverancia y nuestro trabajo: del Trabajo Social. Ante un proyecto neoliberal avasallador, un proyecto ético-político de formación y acción sostenida. 


\section{Referencias}

Aragunde, R. (2019, febrero 28). Hacia dónde podrían dirigirse las universidades de Puerto Rico. Kaosenlared Información contra hegemónica para cambio social. https://kaosenlared.net/hacia-donde-podriandirigirse-las-universidades-de-puerto-rico/

Asociación Nacional de Escuelas de Trabajo Social (ANAETS). (2012). Foro de Formación de Grado. XX Seminario Latinoamericano de Escuelas de Trabajo Social: "Desafíos del Contexto Latinoamericano al Trabajo Social". Córdova, Argentina.

Asociación Nacional de Escuelas de Trabajo Social (ANAETS). (2018). Proyecciones profesionales, académicas y de investigación para el trabajo social en Puerto Rico. Espacios de intercambio y articulación en el marco del XXII Seminario Latinoamericano y Caribeño de Escuelas de Trabajo Social. Bogotá, Colombia.

Basta, R., y Cavalleri, M. (2019).

Formación profesional en Trabajo Social en el actual contexto latinoamericano. Políticas, instituciones y sujetos. Intervención, 9(1), 6-25.

Batalla Plá, M. (2008). E neoliberalismo y su discurso: Desafíos para el Trabajo Social en la realidad contemporánea. Revista Regional de Trabajo Social, 24(42), 49-58.
Chomsky, N. (13 de marzo de 2014). El trabajo académico, el asalto neoliberal a las universidades y cómo debería ser la educación.

SSociólogos. https://ssociologos. com/2014/03/13/noam-chomsky-eltrabajo-academico-el-asalto-neoliberal-alas- universidades-y-como-deberia-ser-laeducación/

Cordero Vega, L. (2011). Las representaciones discursivas que articulan la formación profesional del trabajo social puertorriqueño. Análisis, XII(1), 2-14.

Demetrio Guerra, Y. (2016). La formación profesional en Trabajo Social: proceso de conversación, superación y ruptura en el contexto de América Latina y del Caribe. Revista Costarricense de Trabajo Social, 30, 69-90.

Fontánez Torres, E. (2020). La comunidad política y el derecho a tener derechos. En M. Gómez (Ed.), Derechos y reveses de lo humano (pp. 69-90). Ediciones Laberinto.

Garriga Vidal, L. (2018). El desarrollo de la educación pública primaria y secundaria en Puerto Rico: Vínculos y retos desde el trabajo social escolar. Voces desde el Trabajo Social, 6(1), 174-201. https://doi.org/10.31919/voces.v6i1.127

Hernández Acosta, J. (2019).

Encuesta mundial de valores para Puerto Rico: 2018. Instituto de Estadísticas de Puerto Rico. 
Hernández, C. (26 de mayo de 2019). El fraude educativo. El Nuevo Día. https://www.elnuevodia. com/opinion/columnas/elfraudeeducativocolumna-2495979/

Instituto de Estadísticas de Puerto Rico. (2018). Informe sobre Desarrollo Humano en Puerto Rico: 2016. https://estadisticas.pr/ informe desarrollo humano

Malacalza, S. (2003). La formación del profesional de Trabajo Social en el contexto de la nueva cuestión social en el ámbito institucional. En S.L. Malacalza. Desde el Imaginario Social del Siglo XXI Repensar el Trabajo Social. Espacio Editorial.

Ornelas, A. (2019). Desafíos en la formación universitaria de trabajadores sociales en el contexto de la mercantilización de la educación superior y la fragmentación. En XXII Seminario Latinoamericano y del Caribe de Escuelas de Trabajo Social, Memorias, (pp. 151-161).

Rivera Díaz, M. (2019). La investigación como herramienta socio política para el proyecto de emancipación social desde el Trabajo Social. En XXII Seminario Latinoamericano y del Caribe de Escuelas de Trabajo Social, Memorias, (pp. 93-100).
Román, M. (9 de septiembre de 2016). Evidence based research o el secuestro de un significante. 80 Grados. https://www.80grados.net/ evidence-based-research-o-el-secuestro-deun-significante/

Soto-Román, J. (2019, octubre). Re: Solicitud de colaboración e información [Electronic mailing list message - Ejercicio de exploración entre los programas afiliados a la ANAETS para conocer retos o dificultades en la formación en trabajo social y estrategias o acciones para afrontarlos]. https://docs.google. com/forms/d/e/1FAlpQLSfAAnlhHuWc ZQmJMGWPZs-GjgFBRI YBDGDFGKBn QSf20pumw/viewform 